\title{
Origin of the singular Bethe ansatz solutions for the Heisenberg $X X Z$ spin chain
}

\author{
Jae Dong Noh ${ }^{\mathrm{a}}$, Deok-Sun Lee ${ }^{\mathrm{b}}$, Doochul Kim ${ }^{\mathrm{b}, *}$ \\ a Department of Physics, Inha University, Inchon 402-751, South Korea \\ ${ }^{\mathrm{b}}$ School of Physics, Seoul National University, Seoul 151-742, South Korea
}

Received 11 July 2000

\begin{abstract}
We investigate symmetry properties of the Bethe ansatz wave functions for the Heisenberg $X X Z$ spin chain. The $X X Z$ Hamiltonian commutes simultaneously with the shift operator $T$ and the lattice inversion operator $V$ in the space of $\Omega= \pm 1$ with $\Omega$ the eigenvalue of $T$. We show that the Bethe ansatz solutions with normalizable wave functions cannot be the eigenstates of $T$ and $V$ with quantum number $(\Omega, \Upsilon)=( \pm 1, \mp 1)$ where $\Upsilon$ is the eigenvalue of $V$. Therefore, the Bethe ansatz wave functions should be singular for nondegenerate eigenstates of the Hamiltonian with quantum number $(\Omega, \Upsilon)=( \pm 1, \mp 1)$. It is also shown that such states exist in any nontrivial down-spin number sector and that the number of them diverges exponentially with the chain length. (c) 2000 Published by Elsevier Science B.V. All rights reserved.
\end{abstract}

PACS: $75.10 . J \mathrm{~m} ; 05.50+\mathrm{q}$

Keywords: Heisenberg $X X Z$ spin chain; Singular Bethe ansatz solution; Symmetry

\section{Introduction}

The Bethe ansatz method [1] has been applied to various problems in condensed matter physics. The Heisenberg $X X Z$ spin chain, the Hubbard model, the Kondo model, and many other models are solved by the Bethe ansatz or its variants [2]. The Heisenberg $X X Z$ spin chain describes various physical systems including interacting fermion systems. It is also related to the transfer matrix of the six-vertex model [3], the asymmetric exclusion process, and a Kardar-Parisi-Zhang-type growth model [4]. For the $X X Z$ Hamiltonian, the Bethe ansatz expresses energy eigenvalues and eigenstates as functions of quasi-particle momenta which should satisfy a set of algebraic equations,

\footnotetext{
${ }^{*}$ Corresponding author. Fax: +82-2-884-3002.

E-mail address: dkim@snu.ac.kr (D. Kim).
} 
the Bethe ansatz equations (BAE). Various numerical and analytic methods have been developed to solve the BAE, from which the ground-state energy and low-lying excitation spectrum are calculated [5-10]. Combined with a finite-size-scaling theory, they provide useful tools to study equilibrium and nonequilibrium critical phenomena.

When applying the Bethe ansatz method to the Heisenberg $X X Z$ spin chain, one may doubt whether the Bethe ansatz produces the complete set of eigenstates. The completeness of the Bethe ansatz is proved for an inhomogeneous generalization of the $X X Z$ chain threaded by the Aharonov-Bohm flux $[11,12]$. It implies that the Bethe ansatz for the $X X Z$ Hamiltonian is also complete in a general sense. One may obtain the eigenstate of the $X X Z$ Hamiltonian from that of the generalized Hamiltonian by taking a limit where the inhomogeneity and the flux vanish.

On the other hand, it has also been investigated for a long time whether the Bethe ansatz produces all eigenstates of the $X X Z$ Hamiltonian without the help of the limiting procedure. There have been the arguments for the completeness assuming a string conjecture. For the $X X X$ chain, which has isotropic spin-spin interaction, Takahashi [13] showed that the number of the states constructed from the Bethe ansatz using the string conjecture is equal to the total number of the spin states. The string conjecture was also adopted to prove the completeness of the Bethe ansatz for a generalized $X X Z$ chain [14] and the Hubbard model [15]. Later, the string conjecture was shown to be invalid by Essler et al. [16], who analyzed the algebraic Bethe ansatz equations in the two down-spin sector. They found that some solutions expected from the string conjecture are missing and that there exists solutions violating the string conjecture. Nevertheless, they concluded that the Bethe ansatz is complete at least in the two down-spin sector because the numbers of the former and the latter are the same.

However, one of the solutions found in Ref. [16] is singular; the corresponding Bethe ansatz wave function is not well defined. In fact, the state $|\Psi\rangle=\sum_{l}(-1)^{l}|l, l+1\rangle$, which is an eigenstate of the $X X Z$ Hamiltonian, is not produced from the regular (non-singular) solutions of the Bethe ansatz (see below for the definition of the state $|l, l+1\rangle)$. Quasi-particle momenta corresponding to the state are divergent $[1,17,18]$ and the Bethe ansatz wave function is ill defined. An eigenstate that is not produced from the regular solutions of the Bethe ansatz will be referred to as a singular state. Siddharthan [17] reported that there also exist the singular states in the three down-spin sector. It was claimed that a symmetry property might be important since the singular states have a definite symmetry property.

The singular state does not imply the incompleteness of the Bethe ansatz for the $X X Z$ Hamiltonian. One may obtain the wave functions for the singular states of the $X X Z$ Hamiltonian from those of the generalized Hamiltonian by taking the limit where the inhomogeneity and the flux vanish (see, for example, Ref. [17]). However, it should be examined whether the singular states are relevant to the involved physical quantities of the Heisenberg $X X Z$ spin chain since, if so, one must first consider the generalized model and take the suitable limit $[11,12,17]$. Therefore, it is helpful to answer the question on why there appears the singular states and how many are there in the general down-spin number sectors. In this paper, we investigate the symmetry properties of the 
$X X Z$ Hamiltonian and the Bethe ansatz wave functions to show that the regular solution of the Bethe ansatz with well-defined wave functions does not contain a certain class of eigenstates. Those states are the singular states. We also estimate the number of the singular states approximately at each sector with fixed number of down spins and found that the number of them diverges exponentially with the chain length.

\section{Bethe states and symmetry operations}

The Heisenberg $X X Z$ spin chain of length $N$ has the Hamiltonian

$$
H=-\frac{1}{2} \sum_{l=1}^{N}\left\{\sigma_{l}^{+} \sigma_{l+1}^{-}+\sigma_{l}^{-} \sigma_{l+1}^{+}+\frac{\Delta}{2}\left(\sigma_{l}^{z} \sigma_{l+1}^{z}-1\right)\right\},
$$

where $\sigma_{l}^{x, y, z}$ are the Pauli matrices at site $l, \sigma_{l}^{ \pm}=\left(\sigma_{l}^{x} \pm \mathrm{i} \sigma_{l}^{y}\right) / 2$. The periodic boundary condition is imposed, $\sigma_{N+1}^{x, y, z}=\sigma_{1}^{x, y, z}$. We assume $N$ is even unless stated otherwise. It reduces to the ferromagnetic (antiferromagnetic) $X X X$ chain in the case of $\Delta=1(\Delta=$ $-1)$. Since the Hamiltonian commutes with the magnetization $M=\sum_{l} \sigma_{l}^{z}$, one can work in the $Q$ down-spin sector in the diagonal basis of $\sigma^{z}$. A state in the $Q$ down-spin sector is spanned by ${ }_{N} C_{Q}$ state vectors $\left|x_{1}, x_{2}, \ldots, x_{Q}\right\rangle$ with $1 \leqslant x_{i} \leqslant N\left(x_{i}<x_{j}\right.$ for $\left.i<j\right)$ denoting the position of the $i$ th down spin.

The eigenstate of $H$ can be written as $|\Psi\rangle=\sum_{x_{1}<\cdots<x_{Q}} \psi\left(x_{1}, \ldots, x_{Q}\right)\left|x_{1}, \ldots, x_{Q}\right\rangle$. According to the Bethe ansatz, the wave function is given in terms of quasi-particle momenta $k_{m}(m=1, \ldots, Q)$ by

$$
\psi\left(x_{1}, \ldots, x_{Q}\right)=\sum_{P} A(P) \exp \left(\mathrm{i} \sum_{m=1}^{Q} k_{P_{m}} x_{m}\right),
$$

where the sum is over all permutations $P$ of integers $\{1,2, \ldots, Q\}$. The quasi-particle momenta are complex numbers with $-\pi<\operatorname{Re} k \leqslant \pi$. The amplitudes $A$ 's for different permutations $P$ and $P^{\prime}$, which are identical except for two neighboring integers such that $P_{j}=P_{j+1}^{\prime}=m, P_{j+1}=P_{j}^{\prime}=l$, and $P_{i}=P_{i}^{\prime}$ for $i \neq j, j+1$, are related as

$$
\frac{A(P)}{A\left(P^{\prime}\right)}=-\frac{\mathrm{e}^{\mathrm{i}\left(k_{m}+k_{l}\right)}-2 \Delta \mathrm{e}^{\mathrm{i} k_{m}}+1}{\mathrm{e}^{\mathrm{i}\left(k_{m}+k_{l}\right)}-2 \Delta \mathrm{e}^{\mathrm{i} k_{l}}+1} .
$$

The periodic boundary condition implies the Bethe ansatz equations (BAE) for the quasi-particle momenta:

$$
\mathrm{e}^{\mathrm{i} N k_{m}}=(-1)^{Q-1} \prod_{l \neq m} \frac{\mathrm{e}^{\mathrm{i}\left(k_{m}+k_{l}\right)}-2 \Delta \mathrm{e}^{\mathrm{i} k_{m}}+1}{\mathrm{e}^{\mathrm{i}\left(k_{m}+k_{l}\right)}-2 \Delta \mathrm{e}^{\mathrm{i} k_{l}}+1} .
$$

The state constructed from the Bethe ansatz is called the Bethe state. It has the energy $E=\sum_{m=1}^{Q}\left(\Delta-\cos k_{m}\right)$.

In the special case of $\Delta=0$, where the BAE becomes $\mathrm{e}^{\mathrm{i} k_{m} N}=(-1)^{Q-1}$, the Bethe ansatz produces all eigenstates of $H$. A solution is obtained by selecting $Q$ different values among the $N$ values of $(2 \pi / N) \times$ integers (half-integers) for odd (even) $Q$. There 
are ${ }_{N} C_{Q}$ different ways for each $Q$, which is equal to the possible spin states. Naively, one may think that the solutions at $\Delta=0$ evolve continuously as $\Delta$ is turned on. However, it will turn out to be false.

The $X X Z$ Hamiltonian under the periodic boundary condition commutes with the shift operator $T$, which shifts the position of each down spin to the left by one unit. By construction, the Bethe state is the eigenstate of $T$ with the eigenvalue $\Omega=\left[\prod_{m=1}^{Q} \mathrm{e}^{\mathrm{i} k_{m}}\right]$. Since $T^{N}$ is the identity operator, $\Omega^{N}$ should be unity. The Hamiltonian also commutes with the lattice inversion operator $V$ defined by $V\left|\left\{x_{i}\right\}\right\rangle=\left|\left\{y_{i}\right\}\right\rangle$ with $y_{i}=N-x_{Q-i+1}+1$. Since $V^{2}$ is the identity operator, the eigenvalue of $V$, which will be denoted by $\Upsilon$, takes the value of \pm 1 . The inversion operator does not commute with the shift operator in general. But it is easy to show that they commute with each other in the subspace of $\Omega= \pm 1 ;[T, V]|\Psi\rangle=0$ if $T|\Psi\rangle= \pm|\Psi\rangle$. Then the eigenstate of $H$ can be made as the simultaneous eigenstate of $T$ and $V$ in the subspace.

The Bethe state with $\Omega= \pm 1$ is not necessarily the eigenstate of $V$. If a Bethe state $|\Psi\rangle$ with $\Omega= \pm 1$ with well-defined wave function is a simultaneous eigenstate of $V(V|\Psi\rangle=\Upsilon|\Phi\rangle)$, then one can show that $\Upsilon$ should be equal to $\Omega$ :

$$
\Upsilon=\Omega \text {. }
$$

It is proved as follows. Suppose that $|\Psi\rangle$ is a Bethe state in the $Q$ down-spin sector with the quasi-particle momenta $\left\{k_{1}, k_{2}, \ldots, k_{Q}\right\}$ satisfying Eq. (4). The wave function is given as in Eq. (2). When one applies the lattice inversion operator $V$ to the state $|\Psi\rangle$, the wave function of the state $V|\Psi\rangle=\sum \tilde{\psi}\left(x_{1}, \ldots, x_{Q}\right)\left|x_{1}, \ldots, x_{Q}\right\rangle$ is given by

$$
\begin{aligned}
\tilde{\psi}\left(x_{1}, \ldots, x_{Q}\right) & =\psi\left(N-x_{Q}+1, \ldots, N-x_{1}+1\right) \\
& =\Omega \sum_{P} \tilde{A}(P) \exp \left[\mathrm{i} \sum_{m}\left(-k_{P_{m}}\right) x_{m}\right],
\end{aligned}
$$

where $\tilde{A}(P)=A(P \bar{P})$ with a parity permutation $\bar{P}$ which maps $\{1,2, \ldots, Q\}$ to $\{Q, \ldots, 2,1\}$, and we used that $\left[\prod_{m=1}^{Q} \mathrm{e}^{\mathrm{i} k_{m}}\right]=\Omega$ and $\Omega^{N}=1$. One can easily verify that $\left\{-k_{m}\right\}$ satisfies the BAE in Eq. (4) and that the amplitude $\tilde{A}(P)$ satisfies Eq. (3) with $\left\{k_{m}\right\}$ replaced by $\left\{-k_{m}\right\}$. This shows that $V|\Psi\rangle$ is a Bethe state with the quasi-particle momenta $\left\{-k_{1},-k_{2}, \ldots,-k_{Q}\right\}$. Therefore, a Bethe state could be an eigenstate of $V$ only when the two sets $\left\{k_{1}, k_{2}, \ldots, k_{Q}\right\}$ and $\left\{-k_{1},-k_{2}, \ldots,-k_{Q}\right\}$ are identical.

We rewrite the wave function in Eq. (6), introducing the permutation $\tilde{P}$ defined by the relation $-k_{l}=k_{\tilde{P}_{l}}$, as

$$
\begin{aligned}
\tilde{\psi}\left(\left\{x_{m}\right\}\right) & =\Omega \sum_{P} \tilde{A}(P) \exp \left[\mathrm{i} \sum_{m} k_{\tilde{P} P_{m}} x_{m}\right] \\
& =\Omega \sum_{P} A(\tilde{P} P \bar{P}) \exp \left[\mathrm{i} \sum_{m} k_{P_{m}} x_{m}\right] .
\end{aligned}
$$

Comparing it with Eq. (2), one can find $\Upsilon$ from the ratio of $A(P)$ and $\Omega A(\tilde{P} P \bar{P})$ for a certain permutation $P$, e.g., the identity permutation $I$. If the amplitude $A$ is nonzero 
and finite, $\Upsilon$ is given by

$$
\Upsilon=\Omega A(\tilde{P} \bar{P}) / A(I) \text {. }
$$

Note that the set $\left\{k_{m}\right\}$ is equal to $\left\{-k_{m}\right\}$. On the one hand, unless both 0 and $\pi$ are present in the set $\left\{k_{m}\right\}$, one can rearrange the momenta in such a way that the permutation $\tilde{P}$ and the parity permutation $\bar{P}$ are the same. Then one has $A(\tilde{P} \bar{P})=$ $A\left(\bar{P}^{2}\right)=A(I)$ and hence $\Upsilon=\Omega$. On the other hand, if both 0 and $\pi$ are present in $\left\{k_{m}\right\}, Q$ should be even and one can rearrange the momenta in the following way:

$$
\left\{k_{1}, \ldots, k_{Q / 2-1}, k_{Q / 2}, k_{Q / 2+1},-k_{Q / 2-1}, \ldots,-k_{1}\right\},
$$

with $k_{Q / 2}=0$ and $k_{Q / 2+1}=\pi$. With this arrangement, the permutation $\tilde{P} \bar{P}$ maps $Q / 2$ to $Q / 2+1, Q / 2+1$ to $Q / 2$, and $j \neq Q / 2, Q / 2+1$ to $j$. Then, using Eq. (3), the ratio between $A(I)$ and $A(\tilde{P} \bar{P})$ is given by

$$
\frac{A(I)}{A(\tilde{P} \bar{P})}=-\frac{\mathrm{e}^{\mathrm{i} k_{Q / 2+1}+\mathrm{i} k_{Q / 2}}-2 \Delta \mathrm{e}^{\mathrm{i} k_{Q / 2}}+1}{\mathrm{e}^{\mathrm{i} k_{Q / 2+1}+\mathrm{i} k_{Q / 2}}-2 \Delta \mathrm{e}^{\mathrm{i} k_{Q / 2+1}}+1}=1 .
$$

Therefore, we find that $V|\Psi\rangle=\Omega|\Psi\rangle$ if $|\Psi\rangle$ is an eigenstate of $V$. This proves Eq. (5).

\section{Perturbative construction of singular states}

Eq. (5) shows that the Bethe state with well-defined wave function cannot be the simultaneous eigenstate of $T$ and $V$ with $(\Omega, \Upsilon)=( \pm 1, \mp 1)$. But there exist many eigenstates of $H$ with such symmetry. Suppose an eigenstate of $H$ possesses the quantum numbers $(\Omega, \Upsilon)=(1,-1)$. The state may be degenerate (having the same energy) with another state which has $\Upsilon=+1$ in the same $\Omega$ sector. In the degenerate case, the Bethe state may be a linear superposition of the degenerate pair, which does not lead to the contradiction to Eq. (5). On the other hand, if the eigenstate does not have the degenerate partner with $\Upsilon=1$ in the same $\Omega$ sector, such a state cannot be a Bethe state, hence is absent in the regular Bethe ansatz solutions. In the remaining part, we show that there exist nondegenerate states with $(\Omega, \Upsilon)=( \pm 1, \mp 1)$ for nonzero $\Delta$ in the general $Q$ down-spin sector.

It is convenient to work with the second quantized form of the Hamiltonian. Under the Jordan-Wigner and Fourier transformations, defined as $\sigma_{l}^{+}=a_{l} \exp \left[\mathrm{i} \pi \sum_{j=1}^{l-1} a_{j}^{\dagger} a_{j}\right]$ and $a_{l}=\sum_{p} a_{p} \mathrm{e}^{\mathrm{i} p l} / \sqrt{N}$, respectively, the $X X Z$ Hamiltonian in the $Q$ down-spin sector reads as $H=H_{0}+H_{1}$ where $H_{0}=-\sum_{p}(\cos p-\Delta) n_{p}$ and

$$
H_{1}=-\frac{\Delta}{N} \sum_{p_{1}+p_{2}=p_{3}+p_{4}} \mathrm{e}^{-\mathrm{i}\left(p_{1}-p_{4}\right)} a_{p_{1}}^{\dagger} a_{p_{2}}^{\dagger} a_{p_{3}} a_{p_{4}} .
$$

Here $a_{p}$ and $a_{p}^{\dagger}$ are anticommuting fermion operators with the bare momentum $-\pi<$ $p \leqslant \pi$ and $n_{p}=a_{p}^{\dagger} a_{p}$ is the number operator with $\sum_{p} n_{p}=Q$. The momentum $p$ takes the real value of $(2 \pi / N) \times$ integer (half-integer) for odd (even) $Q$. 


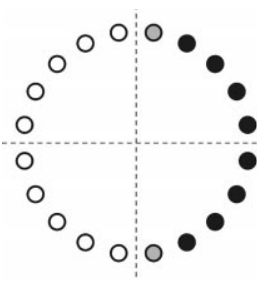

(a)

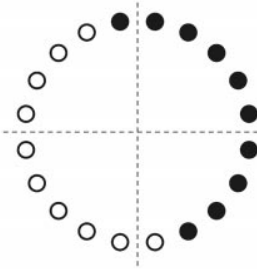

(b)

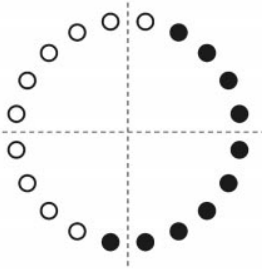

(c)

Fig. 1. An illustration of sets $S_{Q-2}$ and $R$, and a state in Eq. (10) with $N=20$ and $Q=10$. The bare momenta $p$ are denoted by equi-spaced circles in a complex $z \equiv \mathrm{e}^{\mathrm{i} p}$ plane. The horizontal (vertical) dashed lines stand for the real (imaginary) $z$-axis. (a) The elements of the set $S_{Q-2}(R)$ are denoted by black (gray) circles. In (b) and (c) black circles denote the occupied momenta in Eq. (10).

Consider a symmetric set $S_{Q-2}$ which consists of $(Q-2)$ bare momenta satisfying $\sum_{p \in S_{Q-2}} p=0(\bmod 2 \pi)$. A set $S$ is symmetric if it contains $-p$ for all $p \in S$. For a given $S_{Q-2}, R$ is defined as the set of all momenta in the interval $-\pi / 2<p<\pi / 2$ except $p^{\prime}$ or $\pi-p^{\prime}(\bmod 2 \pi)$ for $p^{\prime} \in S_{Q-2}$. Note that $R$ is also a symmetric set. Then, at $\Delta=0$, the following states

$$
\left|p_{\alpha} ; S_{Q-2}\right\rangle \equiv a_{p_{\alpha}}^{\dagger} a_{\pi-p_{\alpha}}^{\dagger} \prod_{p \in S_{Q-2}} a_{p}^{\dagger}|0\rangle,
$$

with $p_{\alpha} \in R$ are degenerate eigenstates of $H$ in the $Q$ down-spin sector having $\Omega=-1$. Here $|0\rangle$ denotes the vacuum, i.e., $a_{p}|0\rangle=0$ for all $p$. They are not the eigenstates of $V$. One can easily show that

$$
V\left|p_{\alpha} ; S_{Q-2}\right\rangle=-\Omega\left|-p_{\alpha} ; S_{Q-2}\right\rangle .
$$

Fig. 1 illustrates an example of the set $S_{Q-2}$ and corresponding $R$ with the states in Eq. (10) for $N=20$ and $Q=10$.

As $\Delta$ is turned on, $H_{1}$ generates the overlap between the degenerate states, which may cause the degeneracy to split. To see the degeneracy splitting, we treat $H_{1}$ as a perturbation and apply the degenerate perturbation theory. In this scheme, the leading order correction to the energy is given by the eigenvalue of the perturbation matrix $\left(H_{1}\right)_{p_{\alpha}, p_{\beta}} \equiv\left\langle p_{\alpha} ; S_{Q-2}\left|H_{1}\right| p_{\beta} ; S_{Q-2}\right\rangle$ with $p_{\alpha}, p_{\beta} \in R$. The eigenstates are given by the eigenvectors of the perturbation matrix. A straightforward calculation shows that the matrix element is given by

$$
\left(H_{1}\right)_{p_{\alpha}, p_{\beta}}=-\frac{4 \Delta}{N}\left(\cos p_{\alpha}\right)\left(\cos p_{\beta}\right),
$$

where we neglect a constant diagonal element. It has the nondegenerate eigenvector

$$
\left|\Psi ; S_{Q-2}\right\rangle=\sum_{p_{\alpha} \in R} \cos p_{\alpha}\left|p_{\alpha} ; S_{Q-2}\right\rangle,
$$

with the eigenvalue $-(4 \Delta / N) \sum_{p_{\alpha} \in R} \cos ^{2} p_{\alpha}$. The other states remain to be degenerate with their common eigenvalue 0 . If one applies $V$ to the state $\left|\Psi ; S_{Q-2}\right\rangle$ and uses Eq. (11), one can see that the nondegenerate state is the eigenstate of $V$ with $\Upsilon=+1$ 
while $\Omega=-1$. Therefore, it cannot be produced from the regular solution of the Bethe ansatz by Eq. (5).

The remaining degenerate states may split further in the higher-order perturbations. So the number of the nondegenerate states with $(\Omega, \Upsilon)=(-1,+1)$ is equal to or greater than the number of different realizations of the set $S_{Q-2}$. For even $Q, S_{Q-2}$ consists of $(Q-2) / 2$ values of $p$ selected randomly in the interval $0<p<\pi$ and their negative partners. For odd $Q, S_{Q-2}$ consists of $p=0,(Q-3) / 2 p$ 's randomly selected in the interval $0<p<\pi$, and their negative partners. So, there are at least ${ }_{N / 2} C_{(Q-2) / 2}\left({ }_{N / 2} C_{(Q-3) / 2}\right)$ states which have $(\Omega, \Upsilon)=(-1,+1)$ and they are those states which cannot be produced from the Bethe ansatz in the even (odd) $Q$ down-spin sector. If we sum over all $Q$, the number of such states is of the order of $2^{N / 2}$.

In the two down-spin sector, the perturbation calculation becomes exact since the states in Eq. (10) with the empty set $S_{Q-2}$ span the whole two down-spin sector with $\Omega=-1$. So the state

$$
\sum_{-\pi / 2<p<\pi / 2}(\cos p) a_{p}^{\dagger} a_{\pi-p}^{\dagger}|0\rangle
$$

is the eigenstate of $H$ at all $\Delta$, which cannot be produced from the Bethe ansatz. If one performs the inverse Jordan-Wigner and Fourier transformations, the state can be written as $\sum_{l}(-1)^{l}|l, l+1\rangle$. This is the example of the singular states mentioned in Ref. [17].

Also consider the states

$$
a_{p_{\alpha}}^{\dagger} a_{p_{\beta}}^{\dagger} a_{\pi-p_{\alpha}}^{\dagger} a_{\pi-p_{\beta}}^{\dagger} \prod_{p \in S_{Q-4}} a_{p}^{\dagger}|0\rangle
$$

with $p_{\alpha}, p_{\beta} \in R$, constructed from a symmetric set $S_{Q-4}$ consisting of $(Q-4)$ momenta satisfying $\sum_{p \in S_{Q-4}} p=0(\bmod 2 \pi)$ and the set $R$ of all momenta in the interval $-\pi / 2<p<\pi / 2$ except $p^{\prime}$ or $\pi-p^{\prime}(\bmod 2 \pi)$ for $p^{\prime} \in S_{Q-4}$. They are degenerate eigenstates of $H$ at $\Delta=0$ with $\Omega=1$. The same perturbation analysis will show that the degeneracy splits for finite $\Delta$ and there appear nondegenerate states with $\Upsilon=-1$. Those states cannot be produced by the Bethe ansatz from Eq. (5) either. There are ${ }_{N / 2} C_{(Q-4) / 2}\left(N_{N / 2} C_{(Q-5) / 2}\right)$ different ways in choosing the set $S_{Q-4}$ for even (odd) $Q$. For each set, there exists at least one state with $(\Omega, \Upsilon)=(1,-1)$. So the total number of such states is again of order of $2^{N / 2}$.

The same analysis can be applied to the states from the symmetric sets $S_{Q-6}, S_{Q-8}, \ldots$, with $\sum_{p \in S} p=0(\bmod 2 \pi)$ and symmetric sets $S_{Q-1}, S_{Q-3}, \ldots$ containing $p=\pi$ and satisfying $\sum_{p \in S}=\pi(\bmod 2 \pi)$. For each case, there are at least a number of order of $2^{N / 2}$ singular states.

\section{Numerical results}

The state counting discussed in the previous section is not exact. States from different $S$ sets may be degenerate at $\Delta=0$. In that case perturbation calculations should be 


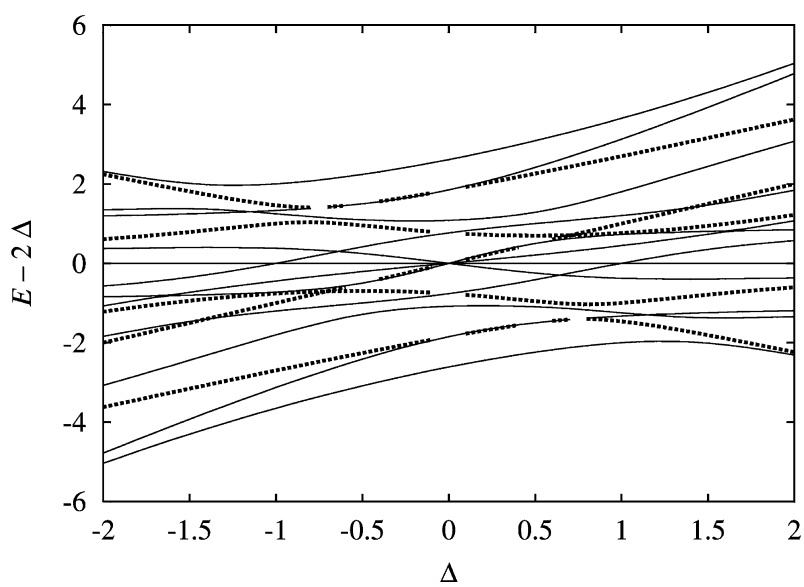

Fig. 2. Energy vs. $\Delta$ with $\Omega= \pm 1$ of the $X X Z$ Hamiltonian in the four down-spin sector with the chain length $N=8$. The dotted lines represent the singular states, nondegenerate eigenstates with quantum number $(\Omega, \Upsilon)=( \pm 1, \mp 1)$.

Table 1

The number of the nondegenerate states with $(\Omega, \Upsilon)=( \pm 1, \mp 1)$

\begin{tabular}{rcrr}
\hline$N$ & $\Delta=0.2$ & 0.5 & 1.0 \\
\hline 8 & 12 & 7 & 12 \\
10 & 25 & 15 & 20 \\
12 & 64 & 29 & 59 \\
14 & 152 & 61 & 152 \\
16 & 339 & 131 & 319 \\
\hline
\end{tabular}

carried out in an enlarged space and it is not guaranteed that each $S$ set generates at least one singular state, and the singular states with $(\Omega, \Upsilon)=( \pm 1, \mp 1)$ may become degenerate accidentally with a state with $(\Omega, \Upsilon)=( \pm 1, \pm 1)$ for a particular value of $\Delta$. Then the Bethe ansatz may produce the linear superpositions of them. We believe that those effects do not affect the leading order of magnitude of the total number of the singular states.

We checked this by diagonalizing the $X X Z$ Hamiltonian exactly with $N=6,8, \ldots, 16$. The symmetry property of each eigenstate is checked and the nondegenerate states with $(\Omega, \Upsilon)=(1,-1)$ and $(-1,1)$ are identified as the singular states. Fig. 2 shows the energy level, as a function of $\Delta$, of each state with $\Omega= \pm 1$ for $N=8$ in the four down-spin sector. The singular states are denoted by dotted lines. Note that each singular state evolves from the degenerate states at $\Delta=0$ as the degeneracy is lifted for nonzero $\Delta$. At $\Delta= \pm \frac{1}{2}$, many singular states become degenerate with non-singular states and they are not counted as the singular states. In Table 1, we show the total number of the singular states at $\Delta=0.2,0.5$, and 1.0. Due to the accidental degeneracy the number varies with $\Delta$. It is confirmed that the number increases approximately as $2^{N / 2}$ at the three values of $\Delta$. 


\section{Discussion and summary}

The singular behaviors of some Bethe ansatz solutions have been noticed for the Heisenberg $X X Z$ model (see, e.g., Refs. [1,5,17]). So the generalizations to the inhomogeneous $X X Z$ model with Aharonov-Bohm flux were considered and their Bethe ansatz solutions were shown to be complete [11,12]. In those cases the Hamiltonian does not possess the lattice inversion symmetry and the present analysis is not applicable.

The singular states include low-lying states in the half-filling case, $Q=N / 2$. Fig. 1(b) and (c) illustrate degenerate first excited states at $\Delta=0$ in the $\Omega=1$ sector. They are not the eigenstates of the inversion operator $V$. For nonzero $\Delta$ the degeneracy is removed, and the states evolve into the eigenstates of $V$ with $\Upsilon= \pm 1$. Therefore, the Bethe ansatz fails to produce one of them with $\Upsilon=-1$. Those states are important in identifying the operator content of the $X X Z$ model in the context of the conformal field theory (see Ref. [5] and references therein). They have the scaling dimension $x_{n, m}$ with $n=0$ and $m= \pm 1$. To recover the whole operator content from the Bethe ansatz, one should introduce the Aharonov-Bohm flux and take the limit where the flux vanishes as done in Ref. [5].

So far, we have assumed $N$ is even. For odd $N$, one cannot find a pair of bare momenta $p$ and $p^{\prime}$ satisfying $p+p^{\prime}=\pi$ and the previous analysis cannot be applied. So the above arguments are not valid for odd $N$, though Eq. (5) holds good. We have performed the same numerical analysis for odd $N \leqslant 15$ and found that the nondegenerate states with $(\Omega, \Upsilon)=( \pm 1, \mp 1)$ do not exist.

In summary, we have shown that the symmetry properties of the Hamiltonian and the Bethe wave functions prevent the regular solutions of the Bethe ansatz from containing some eigenstates. For the fixed number of down spins, the $X X Z$ Hamiltonian can be simultaneously diagonalized with the shift operator $T$ and the lattice inversion operator $V$ in the subspace where the eigenvalue of $T, \Omega$, is \pm 1 , and each simultaneous eigenstate has one of the following four pairs of eigenvalues, $(\Omega, \Upsilon)=$ $(1,1),(-1,-1),(1,-1),(-1,1)$. But there is no regular Bethe ansatz wave function with $(\Omega, \Upsilon)=(1,-1),(-1,1)$, which leads to the appearance of the singular Bethe states. We estimated the number of the singular states as of $O\left(2^{N / 2}\right)$ for even $N$. The number of the singular states is exponentially large though the fraction to the total number of the states, $2^{N}$, is vanishingly small. Moreover, the singular states include low-lying states in the half-filling case where the number of down spins is $N / 2$.

\section{Acknowledgements}

This work was supported in part by the BK21 Project of Ministry of Education, Korea. 


\section{References}

[1] H. Bethe, Z. Phys. 71 (1931) 205.

[2] V.E. Korepin, N.M. Bogoliubov, A.G. Izergin, Quantum Inverse Scattering Method and Correlation Functions, Cambridge University Press, Cambridge, 1993.

[3] R.J. Baxter, Exactly Solved Models in Statistical Mechanics, Academic Press, New York, 1982.

[4] B. Derrida, Phys. Rep. 301 (1998) 65.

[5] F.C. Alcaraz, M.C. Barber, M.T. Bachelor, Ann. Phys. NY 182 (1988) 280.

[6] D. Kim, Phys. Rev. E 52 (1995) 3512.

[7] N.M. Bogoliubov, A.G. Izergin, V.E. Korepin, Nucl. Phys. B 275 (1986) 687.

[8] N.M. Bogoliubov, A.G. Izergin, N. Yu Reshetikhin, J. Phys. A 20 (1987) 5361.

[9] F. Woynarovich, H.-P. Eckle, J. Phys. A 20 (1987) L97.

[10] J.D. Noh, D. Kim, Phys. Rev. E 53 (1996) 3225.

[11] R.P. Langlands, Y. Saint-Aubin, preprint.

[12] V. Tarasov, A. Varchenko, Int. Math. Res. Notices 13 (1995) 637.

[13] M. Takahashi, Prog. Theo. Phys. 46 (1971) 401.

[14] A.N. Kirillov, N.A. Liskova, J. Phys. A 30 (1997) 1209.

[15] F.H.L. Essler, V.E. Korepin, K. Schoutens, Phys. Rev. Lett. 67 (1991) 3848.

[16] F.H.L. Essler, V.E. Korepin, K. Schoutens, J. Phys. A 25 (1992) 4115.

[17] R. Siddharthan, cond-mat/9804210.

[18] A. Wal, T. Lulek, B. Lulek, E. Kozak, Int. J. Mod. Phys. B 13 (1999) 3307. 